\title{
Bronchoscopic removal of foreign bodies in adults: experience with 62 patients from 1974-1998
}

\author{
A. Debeljak, J. Šorli, E. Mušič, P. Kecelj
}

Bronchoscopic removal of foreign bodies in adults: experience with 62 patients from 19741998. A. Debeljak, J. Sorli, E. Mušic, P. Kecelj. C ERS Journals Ltd 1999.

ABSTRACT: The authors reviewed their experience with therapeutic bronchoscopy for removal of tracheobronchial foreign bodies in the adult.

Bronchoscopy records and collection of foreign bodies in the endoscopic department were retrospectively examined. Among 37,466 bronchoscopies performed between 1974-1998, $62(0.2 \%)$ were performed for the removal of tracheobronchial foreign bodies.

Medical history was suggestive of foreign body aspiration in 33 patients and the chest radiograph was suggestive in 10 patients. The procedure was performed with the flexible bronchoscope in 42 patients $(68 \%)$, rigid bronchoscope in $4(6 \%)$, and with both in $16(26 \%)$ patients. Foreign bodies were found in the right bronchial tree on 42 occasions, in the left on 20 and in the trachea once. In 39 patients, inflammatory granulations were found around the foreign body. The origins of the foreign bodies included: bone fragments $(n=31)$, vegetable $(n=10)$, broncholith $(n=8)$, a part of dental prosthesis $(n=7)$, endodontic needle $(n=2)$, a metallic $(n=2)$, or plastic $(n=1)$ particle, a tracheostomy tube $(n=1)$ and a match $(n=1)$. In one patient, 2 foreign bodies were found. The foreign bodies were successfully removed in all but 2 patients $(3 \%)$. The most useful instruments for removal were alligator forceps and the wire basket.

Foreign bodies in the tracheobronchial system are rare in adults. They can be successfully removed in the majority of patients under either flexible or rigid bronchoscopy.

Eur Respir J 1999; 14: 792-795.

Aspiration of foreign bodies can occur in children [1], as well as in adults or elderly people [2]. Therapeutic bronchoscopy for foreign bodies removal was introduced into clinical practice 100 yrs ago by the German otolaryngologist G. Killian [3]. Currently both, rigid and flexible bronchoscopies are utilized for this indication [4].

In the present study, the authors reviewed their experience with therapeutic bronchoscopy for the removal of foreign bodies from 1974-1998. More specifically, diagnosis, techniques of removal and types of foreign bodies present in the tracheobronchial tree were investigated.

\section{Patients and methods}

The authors' Clinical Department for Respiratory Diseases and Allergy covers a population of one million inhabitants in Slovenia. Bronchoscopic records and the collection of foreign bodies in the endoscopic department were retrospectively examined. If possible, hospital documen-tation of the patients with foreign bodies aspiration was reviewed. Between 1974-1998, 37,466 bronchoscopies were performed, 33,716 (90\%) with the flexible and $3,750(10 \%)$ with the rigid bronchoscope. Sixty-two bronchoscopies (i.e. $0.2 \%$ ) were performed in an attempt to remove foreign bodies.

There were 42 male and 20 female patients ranging in age $18-86$ yrs (mean \pm SD $55 \pm 14$ yrs). Prior to broncho-
Clinical Department for Respiratory Diseases and Allergy Golnik, SI-4204 Golnik, Slovenia

\section{Correspondence: A. Debeljak}

Clinical Dept for Respiratory Diseases and Allergy Golnik

SI-4204

Slovenia

Fax: 38664469117

Keywords: Bronchoscopy foreign bodies lung diseases

Received: January 151998

Accepted after revision June 91999 scopy, a complete medical history, physical examination and chest radiograph at full inspiration were obtained. Premedication included $1 \mathrm{mg}$ of atropine. Topical anaesthesia (nose, pharynx and larynx) was performed with $4 \%$ lidocaine and $2 \%$ lidocaine transtracheally, or while introducing the instrument. The authors added $0.1 \mathrm{mg}$ fentanyl in the 20 patients in whom rigid bronchoscopy was performed. A rigid Storz 10318B (Storz, Tuttlingen, Germany) and flexible Olympus 5 BF3, 1T 30 (Olympus, Tokyo, Japan) bronchoscopes were utilized.

\section{Results}

In this retrospective study, the diagnosis of foreign body was suspected prior to bronchoscopy in 34 patients (55\%) from their medical history. In 24 patients, aspiration occurred $>1$ week before bronchoscopy, and in 10 patients, within the week before admission. The longest time between penetration of a foreign body into the lung and admission was 27 yrs and occurred with a metallic fragment.

The radio-opaque nature of the foreign body was confirmed by chest radiograph in 10 patients. The chest radiograph showed pneumonia or pleuro-pneumonia in 34 patients, atelectasis in 12 , localized scaring in 4 , or bronchiectatic changes in 2 . In 9 patients more than one change was found. Nine patients had a normal chest radiograph. 
In 28 patients in whom the time of aspiration could not be ascertained from their medical history, the presumptive diagnosis (before bronchoscopy could be utilized to confirm any diagnosis) was bronchial tumour $(n=14)$, pneumonia $(n=5)$, inactive pulmonary tuberculosis $(n=4)$, bronchiectasis $(n=2)$, recurrent pneumonia $(n=2)$, and pulmonary aspergillosis $(\mathrm{n}=1)$.

The rigid bronchoscope was used in $4(6 \%)$ patients, the flexible bronchoscope in $42(68 \%)$ and both in $16(26 \%)$. In patients in whom both instruments were used, flexible bronchoscopy was performed first in order to explore the tracheobronchial tree. Then, the foreign body was removed via the rigid bronchoscope. The flexible instrument was introduced through the rigid instrument in 5 patients, through an orotracheal tube in 35 patients, via a mouthpiece in 8 and through the naris in 10 patients.

Type of foreign bodies removed (table 1). The most common foreign bodies were pieces of bone. Iatrogenic foreign bodies included endodontic needles and a plastic tracheostomy tube. Foreign bodies of vegetable origin included cherry stones, pea grains, bean, fruit of Arctium lappa, apple pip and thorn.

Location of foreign bodies: the foreign bodies were located in the trachea in 1 patient $(1 \%)$, in the right bronchial tree in $42(67 \%)$ and in the left bronchial tree in 20 patients $(32 \%)$. Twenty-two foreign bodies $(35 \%)$ were in the right lower lobe bronchi (fig. 1).

Underlying mucosal changes: the bronchoscopic appearance of the bronchial mucosa around the foreign body (as examined after removal) was normal in 7 patients. In the remainder of the patients, either granulations $(n=40)$, inflammation $(n=11)$ and scarring $(n=5)$ were seen. In 1 patient who was a smoker, the incidental finding of granulations of squamous cell lung carcinoma was observed in the right bronchus intermedius, while a piece of bone was removed from the anterobasal bronchus of the left lower lobe (LB8).

Removal tools and procedure: foreign body forceps with $(n=11)$, or without optics $(n=6)$ and biopsy forceps $(n=2)$ were used through the rigid bronchoscope. Through the flexible instruments, the authors utilized alligator forceps $(n=15)$, three-teethed forceps $(n=12)$, the wire basket $(n=5)$ and biopsy forceps $(n=2)$. In 3 patients, the foreign body was removed by simple suction. In 4 patients, the foreign body was dislodged by endoscopic instruments, and ex-

Table 1. - Type of foreign body $(n=63)$

\begin{tabular}{ll}
\hline Foreign body & $\mathrm{n}$ \\
\hline Bone & 31 \\
Broncholith & 8 \\
Tooth & 7 \\
Cherry stone & 4 \\
Pea grain & 2 \\
Endodontic needle & 2 \\
Metallic fragment & 1 \\
Tracheostomy tube & 1 \\
Plastic object & 1 \\
Metallic skewer & 1 \\
Bean & 1 \\
Apple pip & 1 \\
Thorn & 1 \\
Fruit of Arctium lappa & 1 \\
Match & 1
\end{tabular}

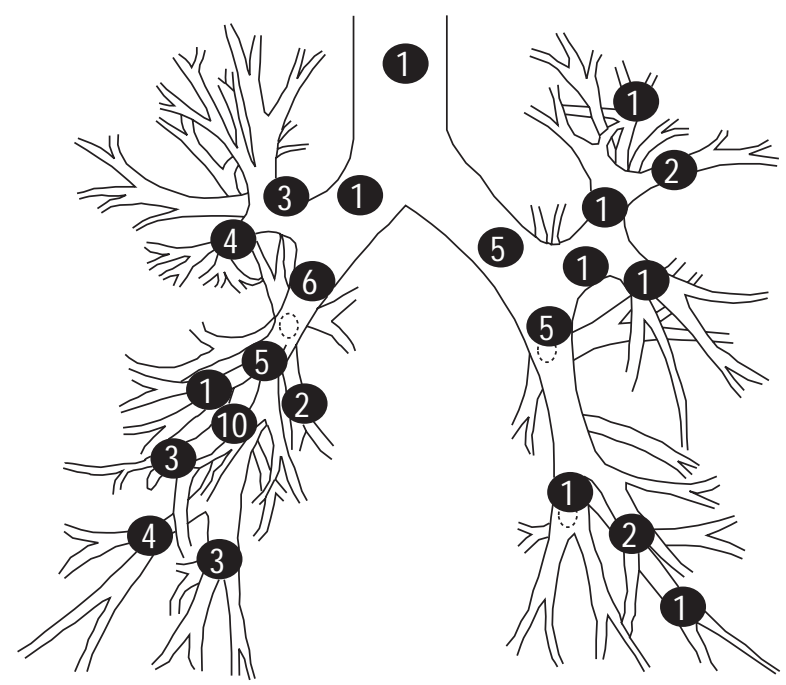

Fig. 1. - Location of 63 foreign bodies in the tracheobronchial tree, in 62 patients. There were a total of 42 foreign bodies found in the right lung and 20 in the left lung.

pectorated immediately after the procedure. In 1 patient, two foreign bodies were removed with the same instrument. The authors were able to remove 61 of the 63 (i.e. $97 \%$ ) of foreign bodies discovered.

The only two failures of the approach for removal of foreign bodies were a piece of bone in the apical bronchus of the right lower lobe (RB6) and a fragment of metal in the right middle lobe bronchus. In the first patient, the bone was left in place without any consequence at the time of this writing. The second patient was wounded into the right side of the chest during an explosion in an iron foundry. A piece of metal penetrated the right lung. After $27 \mathrm{yrs}$, the patient was admitted to the hospital because of pneumonia of the right middle lobe. A chest radiograph and flexible bronchoscopy confirmed that a piece of metal was located into the right middle lobe bronchus. Attempt at removal with both types of bronchoscopes was unsuccessful. Surgical resection of the right middle lobe was performed.

In 8 patients in whom the first bronchoscopy in another institution had failed, the authors successfully removed four foreign bodies with a rigid and four with a flexible bronchoscope. In these eight cases, success was achieved with the type of bronchoscope that had not been utilized before. Illustrative examples include: 1) during dental care, the patient inhaled the endodontic needle. A chest radiograph confirmed its location in the posterobasal bronchus of the right lowr lobe (RB10). Attempt at removal with the rigid bronchoscope at another institution had failed. The authors performed a flexible bronchoscopy, and successfully removed the needle by means of alligator forceps. The large forceps of the rigid bronchoscope could obviously not be introduced and opened in the narrow segmental bronchus in which the needle had located, while the tiny alligator forceps could grasp and remove it. 2) At another institution, a bone in the left main bronchus was diagnosed by flexible bronchoscopy. Removal of the bone was unsuccessful because the jaws of flexible bronchoscope slipped repeatedly and the foreign body could not be grasped. A rigid bronchoscopy was performed by the authors and the bone was removed with alligator forceps at the first attempt. 3) A gold dental crown that was found in the left 
lower lobe bronchus in one patient was removed with the rigid alligator forceps smoothly, after it was rotated with the hollow side proximally.

A special approach was required for patients with tracheostomy. In one patient the inner part of the tracheostomy tube broke and lodged into the right mainstem bronchus. Both flexible and rigid bronchoscopes were required for successful removal.

\section{Discussion}

In the last 24 yrs, tracheobronchial foreign bodies were rare in the authors' practice. The largest series of adults with foreign bodies (60 cases) in recent years emanated from the Mayo Medical Center [5]. Age and the male/ female ratio in the current series (age $55 \mathrm{yrs}$, male/female ratio 2.1:1) were similar to those in the Mayo Medical Center series (age 60 yrs, male/female ratio 2.4:1). In children, the incidence of tracheobronchial foreign bodies is characteristically higher than in adults [6].

The diagnosis of foreign bodies is difficult to establish in patients with a noncharacteristic medical history and discrete symptoms [7]. Accordingly, the authors were able to make a presumptive diagnosis of foreign body before bronchoscopy in only $55 \%$ of patients. This contrasts with $100 \%$ of children, in whom the suspicion of foreign body aspiration existed when referred to the hospital, according to MARTINOT et al. [8]. Possibly, more evident choking in children is an explanation.

Unlike in children, clinical presentation of foreign bodies in adults commonly occurs without asphyxia and is therefore suggestive of chronic lung disease, for instance bronchial tumours, or pneumonia. In only 2 of the current patients $(3 \%)$ was haemoptysis present, while others have observed haemoptysis in $\leq 15 \%$ [5].

The chest radiograph was diagnostic in only $10(14 \%)$ of the patients (only in those with opaque foreign body). This is in accordance with the data in children, in whom a definitely radio-opaque shadow was seen in only $20 \%$ of the cases [8]. Pneumonic and atelectatic radiographic changes were found in $46(74 \%)$ of the current patients, regardless of the time that had elapsed after the foreign body aspiration. The experience of the authors is similar to that by LAN [9], as in $72 \%$ of those patients, the chest radiograph showed a pneumonic infiltrate. At variance with others [5], the current authors feel that a chest radiograph with atelectasis or pneumonia without history of aspiration is not especially suggestive of tracheobronchial foreign body in adults. Like LAN [9] the authors think, that chest radiograph is nonspecific in most adult patients with prior foreign body aspiration. In any event, atelectasis, pneumonia (especially if recurrent) and localized bronchiectasis should suggest the diagnosis.

As in the series of JACKSON and JACKSON [10], the current series showed the majority of foreign bodies to be in the right bronchial tree, especially bronchi of the right lower lobe. The location was predominantly in segmental and lobar bronchi.

In the present series, the most frequent foreign bodies were bones. It is thought that reason for this is that in Slovenia meat dishes usually contain bones. This observation is comparable with the experience of LAN [9], who found bones in $49 \%$ of patients. None of the current patients aspirated bone because of neurological disorders or loss of consciousness, conditions observed by other authors [5]. The bones and some vegetable foreign bodies were inhaled during eating, while some foreign bodies, like thorn and a match were used as toothpicks, as also described by others [9]. Another possibility is to inadvertently inhale a piece of plant, when sucking and chewing vegetable material [11]. Parts of denture were often removed in the patients. None was aspirated in the present series after facial trauma, as observed by others [12]. A plastic object in an 18-yr old patient, was a part of a pencil. Aspirated school supplies may be detected in children and adolescents [13]. The largest foreign body in the patients was the broken inner plastic part of a tracheostomy tube. This kind of foreign body is rather rare [14]. Since the authors successfully removed two endodontic needles from segmental bronchi with the flexible bronchoscope, they agree with other authors that flexible bronchoscopy may be superior to rigid bronchoscopy for grasping tiny and far reaching foreign bodies [15]. A long-standing foreign body can move into bronchus by migration and endobronchial erosion from the lung parenchyma [16], such as that observed in one of the current patients (i.e. a piece of metal in the middle lobe bronchus). This required surgical removal.

It was preferred to introduce the flexible bronchoscope through the orotracheal tube because small foreign bodies can be removed more safely in this manner. It is less likely that the foreign body will escape from the forceps when dragged through a tube than through the pharynx, nose or mouth.

The authors were able to remove tracheobronchial foreign bodies in $97 \%$ of patients utilizing both types of bronchoscopes. Others, who successfully removed $95 \%$ [5] of foreign bodies using the rigid bronchoscope only, obtained similar results. It is agreed that both flexible and rigid bronchoscopy should be used for the diagnosis and removal of foreign bodies in adults [17]. Like PRAKASH et al. [18], the present authors are convinced that flexible and rigid bronchoscopes are complementary tools. Flexible bronchoscopy was successful in $68 \%$ of the patients. The experience of the present authors was similar to that of others, who were able to remove foreign bodies with the flexible bronchoscope in $>90 \%$ of patients [19]. In children who have a narrower tracheobronchial tree than adults, and who may have central, asphyxiating foreign bodies, the rigid bronchoscope under general anaesthesia is preferentially indicated. A good working relationship among pneumonologist, otolaryngologist and thoracic surgeon should exist, in order to effectively manage the patient with a suspected foreign body [8].

\section{Conclusion}

In order to prevent the aspiration of foreign bodies, it is recommended not to put nuts or bones into the mouth and not to keep toothpicks, school supplies and similar items in the mouth. Since a diagnostic suspicion of tracheobronhial foreign body was lacking in half of the adults seen, the authors performed diagnostic bronchoscopies with the flexible bronchoscope. If a foreign body is found, then the authors may remove it with a flexible instrument, especially if it is small or located in a peripheral bronchial 
tube. When the flexible bronchoscopy fails, the authors then rely on the rigid bronchoscope, especially if the foreign body is large and located in the central bronchi and trachea.

\section{References}

1. Hughes CA, Baroody FM, Marsh BR. Pediatric tracheobronchial foreign bodies: historical review from the Johns Hopkins Hospital. Ann Otol Rhinol Laryngol 1996; 105: 555-561.

2. Lamaze R, Trechot P, Martinet Y. Bronchial necrosis and granuloma induced by the aspiration of a tablet of ferrous sulphate. Eur Respir J 1994; 7: 1710-1711.

3. Killian G. Meeting of the Society of physicians of Freiburg. Dec 17, 1897. München Med Wschr 1898; 45: 378.

4. Bolliger CT. Interventionelle Bronchoskopie. Schweiz Rundsch Med Prax 1994; 83: 1378-1382.

5. Limper AH, Prakash UBS. Tracheobronchial foreign bodies in adults. Ann Intern Med 1990; 112: 604-609.

6. Forte V, Gaffney R. Bronchoscopy in the pediatric age group: An ear-nose-throat surgeon's experience. Adv Pediatr Pulmonol 1997; 111: 105-111.

7. Pirozynski M, Zaleska J, Polubiec-Kownacka M. Zastosowanie bronchofibroskopii $\mathrm{w}$ usuwaniu cial obcych $\mathrm{z}$ dolnych drog oddechowych. Pneumonol Alergol Polska 1994; 62: 254-259.

8. Martinot A, Closset M, Marquette CH, et al. Indications for flexible versus rigid bronchoscopy in children with suspected foreign body aspiration. Am J Respir Crit Care Med 1997; 155: 1676-1679.
9. Lan PS. Non-asphyxiating tracheobronchial foreign bodies in adults. Eur Respir J 1994; 7: 510-514.

10. Jackson C, Jackson CL. Foreign bodies in the air and food passages. In: Diseases of the nose, throat, and ear. 2nd ed. Philadelphia, London, WB Saunders, 1959; 849-855.

11. Anonymus. Case Records of the Massachusetts General Hospital - Case 33. N Engl J Med 1997; 337: 1220-1226.

12. Tal-Or E, Schwarz Y, Bloom Y, Kluger Y, Roodik V, Sorkin P. Aspirated tooth removal from airway through tracheotomy and flexible bronchoscopy. J Trauma 1996; 40: 1929-1930.

13. Darrow DH, Holinger LD. Aerodigestive tract foreign bodies in the older child and adolescent. Ann Otol Rhinol Laryngol 1996; 105: 267-271.

14. Bhattacharjee N. Fractured tracheostomy tubes: 3 case reports. Bangladesh Med Res Council Bull 1994; 20: 8 11.

15. Shabb B, Taha AM, Hamada F, Kanj N. Straight pin aspiration in young women. J Trauma 1996; 40: 827-828.

16. Saunders MS, Cropp AJ, Awad M. Spontaneous endobronchial erosion and expectoration of a retained intrathoracic bullet: case report. J Trauma 1992; 33: 909-911.

17. Uri N, Rivlin J, Greenberg E. Flexible bronchoscopy in the diagnosis of foreign body aspiration. Harefuah 1995; 128: 536-537.

18. Prakash UBS, Midthun DE, Edell ES. Indications for flexible versus rigid bronchoscopy in children with suspected foreign-body aspiration. Am J Respir Crit Care Med 1997; 155: 1017-1019.

19. Donado Una JR, de Miguel Poch E, Casado Lopez ME, Alfaro Abreu JJ. Fiber optic bronchoscopy in extraction of tracheo-bronchial foreign bodies in adults. Arch Bronchopneumol 1998; 34: 76-81. 